\title{
A Survey of Professionally Qualified Physiotherapist Working in Neonatal and Paediatric Intensive Care Units in Punjab
}

\author{
Kumar $^{1}$, Ashok, Shergill ${ }^{2}$, Navkaran \& Jairaman ${ }^{3}$ \\ ${ }^{1}$ Assistant Professor, Department of Sports Science, Punjabi University Patiala (Punjab) India \\ ${ }^{2}$ Ph.D. Student, Dept. Sports Science, Punjabi University Patiala (Punjab) India, \\ mail:navkaran9999@gmail.com. \\ ${ }^{3}$ Assistant Professor, Department of Physiotherapy, Punjabi University Patiala (Punjab) India
}

\section{Abstract}

The purpose of this study was to observe the practice of physiotherapy in Neonatal and Paediatric Intensive Care Units (NICU and PICU) in Punjab state. The design of the study was exploratory cross sectional survey. Data was collected with the help of standardized questionnaire, which was sent to one hundred thirty therapists who worked in thirty eight different hospitals in Punjab and these hospitals have claimed physiotherapy facilities in their NICU and PICU premises. A period of two weeks was given to the therapists for the completion of questionnaire. If the therapists were not able to send the filled questionnaire within two weeks, then a reminder call was given to them for the next two consecutive weeks. It was found that only eighty-four (i.e. 64.61\%) completed questionnaires was received from the twenty five hospitals. Results revealed that $60 \%$ of hospitals were having physiotherapists and out of which $51.2 \%$ therapists were graduate in nursing (B.Sc.), $41.7 \%$ graduate in physiotherapy (BPT) and 7.1\% Master in Physiotherapy (MPT). It was concluded that not all of the patients get routine physiotherapy from the professionally qualified physiotherapists as part of their NICU and PICU stay in hospitals located in Punjab.

Key words: ICU, Neonatal, Paediatric, Physiotherapy, Punjab

\section{Introduction}

Recent advances in medical care have improved the survival of newborn babies born with various problems. Despite this death in the neonatal intensive care unit (NICU) is an inevitable reality. In 1960, the idea of having a special intensive care unit for newborns, a neonatal intensive care unit represented a developmental milestone for the field of neonatology. Neonates as young as 25 weeks and as small as $750 \mathrm{~g}$ are routinely being saved. However, survivors often have significant physical and mental impairments, including cerebral palsy, blindness, and learning disabilities (Manjiri et al., 2011). The infant mortality rate (IMR) in India has dropped from 144 per thousand live births to 84 per thousand live births in the past few decades. In Punjab according to the 2011 census infant mortality rate is 38 per thousand live births. The recommendation is for 30 Neonatal intensive care units beds per million population (Fernandez and Mondkar, 1993). Neonatal chest physiotherapy has become a routine method of care in neonatal intensive care units in western world (Bruno \& Claude, 2007), while the same is less common in India, probably due to shortage of cardiopulmonary physiotherapy training in pediatrics and neonates.NICU is a unit that provides high quality skilled care to critically ill neonates 
by offering facilities for continuous clinical, biochemical and radiological monitoring and use of life support systems with the aim of improving survival of babies (Fernandez and Mondkar, 1993). NICU is typically directed by one or more neonatologists and staffed by nurses, nurse practitioners, pharmacists, physician assistants, resident physicians, and respiratory therapists (Whitfield et al, 2004). Pediatric intensive care unit is an area within a hospital specializing in the care of critically ill infants, children, and teenagers. A PICU is typically directed by one or more pediatric intensivists or PICU consultants and staffed by doctors, nurses, and respiratory therapists who are specially trained and experienced in pediatric intensive care. The unit may also have nurse practitioners, physician assistants, physiotherapists, social workers, child life specialists, and clerks on staff although this varies widely depending on geographic location. The ratio of professionals to patients is generally higher than in other areas of the hospital, reflecting the acuity of PICU patients and the risk of life-threatening complications (Pronovost et al, 2001).

Physiotherapy is sought when there is excess secretion, poor gas exchange, and increased work of breathing or radiologic evidence of atelectasis (Vijay et al., 2012). Children should be handled with care and respect, most physiotherapy techniques used in adults can be applied in children and the same contraindications apply. The role of physiotherapist in the NICU has been associated with the care of neonate lung. Postural drainage with percussion, vibration and suction are incorporated in physiotherapy management. In some institutions around the world extubations are also performed by physiotherapists in accordance with the neonatal unit protocol. In addition to respiratory care, the physiotherapists are also engaged in assessment and management of neonates with either neurological or musculoskeletal disorders (Bertone, 1988). The role of physiotherapist in the neonatal intensive care unit and pediatric intensive care units are positioning, postural drainage, percussion, vibration, suctioning, breathing exercises, forced expiratory techniques, application of aerosols, nebulisation and bronchodilators (Robyn and Robyn, 2003). The basic therapeutic principles in paediatric chest physiotherapy techniques (CPT) are identical to those applied in adults. However, the child's growth and development results in continuing changes in respiratory structure and function and the requirement for different applications of CPT in each age group (Zach and Oberwaldner, 1999). The objectives of CPT are to prevent or reduce the mechanical consequences of obstructing secretions, such as hyperinflation, atelectasis, maldistribution of ventilation, ventilation/perfusion mismatch and increased work of breathing. Another therapeutic concept focuses on removing infective material, inflammatory mediators, and proteolytic and oxidative activity from the airways and in doing so reduces or even prevents host-mediated inflammatory tissue damage (Zach and Oberwaldner, 1987). The most common referred conditions for physiotherapy are hyaline membrane disease (HMD), meconium aspiration, pneumonia, and surgery involving the thorax and abdomen. 
The most common cause of respiratory distress in the neonate is HMD which is related to insufficient levels of surfactant in the lung (Farrell and Avery, 1975). The physiotherapy techniques are beneficial in patients with pneumonia and compromised airway clearance, especially in the clearing stages of infectious process. The purpose of this study was to find whether the NICU and PICU located in Punjab state provide physiotherapy facilities to the intensive neonates and paediatric populations.

\section{Material and Methods:}

The design of the present study was exploratory cross sectional survey. The participants of the present study were physiotherapists and nurses who were working in NICU and PICU.They were requested to fill the questionnaire. A list of hospitals of Punjab state was obtained from the website of Govt. of Punjab, Department of Family and Health Welfare Society and also from various paediatricians across the state. Following which data collection was started from various hospitals and nursing homes in Punjab. The hospitals in Patiala district and nearby towns were personally visited by the investigator and data was collected from the therapists who were worked in NICU and PICU. A questionnaire to the hospitals located in other districts of Punjab was sent either by e-mails or personally by fellow colleagues. The investigator did not get the opportunity to meet every therapist personally. Two weeks time was given to the respondents to fill the questionnaire and after that a reminder call was given to them after consecutive one week. The aim and objectives of the study were clearly stated in a cover letter attached to the questionnaire in order to obtain the consent of respondents. The respondents were made clear that the information gathered from them shall remain confidential and be used only for research purpose. Some therapists filled the questionnaire immediately whereas others asked the investigator to leave the blank questionnaire and collect the filled one at a later date. The investigator distributed the questionnaire to 130 therapists. Repeated requests were made to them regarding to fill the questionnaire two times after 2 weeks. Even after repeated requests made to the respondents, 46 questionnaires was not obtained and only 84 filled questionnaires were collected. Thus the response rate of this survey was $64.61 \%$.

Data analysis- Data was summarized using percentages and tabular forms and graphs.

\section{Results \& Discussion}

A total of 84 completed questionnaires were received out of 130 (64.61\% response rate) with largest response from Patiala, Mohali, Bathinda, Ludhiana, Muktsar in descending order.

Table 1. Hospitals with $\&$ without physiotherapists

\begin{tabular}{lcc} 
Hospitals & N & $\%$ \\
\hline with physiotherapists & 15 & $60 \%$ \\
without physiotherapists & 10 & $40 \%$ \\
\hline
\end{tabular}

Table 1 shows that the number and percentage of hospitals with and without physiotherapists, $15 \quad(60 \%)$ hospitals have permanent physiotherapists in their facility and 10(40\%) were not having any physiotherapists in their facility. 
Table 2. Therapists involved in initial ventilator parameters

\begin{tabular}{ll|l}
\hline $\begin{array}{ll}\text { Involvement in setting initial } \\
\text { ventilator parameters }\end{array}$ & $\mathrm{N}$ & $\%$ \\
\hline Regularly & 16 & $19.05 \%$ \\
Often & 19 & $22.62 \%$ \\
Occasionally & 24 & $28.57 \%$ \\
Never & 25 & $29.76 \%$ \\
\hline
\end{tabular}

Table 2 shows that how often the therapists were involved in setting initial ventilator parameters. It was found that $29.76 \%$ therapist never involved in ventilator settings, $28.57 \%$ were occasionally involved and only $19.05 \%$ therapists were regularly involved in setting of initial ventilator setting. Table 3 show that $45.24 \%$ therapists were never involved in weaning, $21.43 \%$ were regularly involved and $14.29 \%$ were occasionally involved in weaning from mechanical ventilator.

Table 3.Therapists involved in weaning from mechanical ventilator

\begin{tabular}{lcc}
\hline Involved in weaning & $\mathrm{N}$ & $\%$ \\
\hline Regularly & 18 & $21.43 \%$ \\
Often & 16 & $19.04 \%$ \\
Occasionally & 12 & $14.29 \%$ \\
Never & 38 & $45.24 \%$ \\
\hline
\end{tabular}

Table 4 shows that the academic qualifications of the therapist providing physiotherapy treatment in different hospitals. Therapists with B.Sc. nursing $(51.2 \%)$ were mostly indulged in physiotherapy treatment than the qualified professionally qualified physiotherapists with bachelor and masters qualification in physiotherapy $41.7 \%$ and $7.1 \%$ respectively.

Table 4.Table showing qualification of the individuals providing physiotherapy treatment

\begin{tabular}{lcc}
\hline Qualification & $\mathrm{N}$ & $\%$ \\
\hline BPT & 35 & $41.7 \%$ \\
MPT & 6 & $7.1 \%$ \\
PhD & 0 & 0 \\
Diploma & 0 & 0 \\
B.Sc nursing & 43 & $51.2 \%$ \\
\hline
\end{tabular}

\section{Discussion}

A total number of 48 hospitals were identified in Punjab with NICU and PICU facilities and 38 hospitals were invited for the study, out of which 25 responded. The total number of hospitals having physiotherapy facilities was (15) $60 \%$ and (10) $40 \%$ were without the physiotherapy facilities in Punjab. The investigator found very little or no considerable data regarding the role of Physiotherapy in NICU and PICU in India or any of its state and this study may be the first to report the practice of physiotherapy in NICU and PICU in Punjab state. Norrenberg et al, 2000, reported that 38\% of hospital in Europe had more than 30 physiotherapists working in the hospital, but $25 \%$ had no exclusive ICU therapists. $34 \%$ were available during night, $85 \%$ during the weekend. In our study out of 25 hospitals 19 (76\%) hospitals were having teaching facilities and 6 (24\%) hospitals were without teaching facilities. In another study by Lewis et al, 1992, concluded that, methods of chest treatment and the indicators for commencing chest treatment were similar throughout NICU in Australia. Both physiotherapists and nursing staff played a role in chest physiotherapy in all units but in one unit it was the responsibility of the nursing staff. $\mathrm{He}$ reported that in $36 \%$ of units physiotherapists were only providers of the respiratory therapy and 55\% of units shared respiratory therapy between nurses and physiotherapists. In our study the qualification of the individuals providing physiotherapy treatment was also similar in many aspects with the study of Robyn \& Robyn (2003), which reported that 
Respiratory therapy was carried out by physiotherapists and nurses in $54.5 \%$ of units, by physiotherapists only in $36.4 \%$ of units, and by nurses only in the remaining $9.1 \%$ of units surveyed. In our study (43) $51.2 \%$ nurses were involved in providing physiotherapy treatment and (35) $41.7 \%$ of physiotherapists were involved in providing physiotherapy care. Only (6) $7.1 \%$ postgraduate students were involved in providing treatment in NICU and PICU. Jithendra et al. (2007), in his study found that therapists were not involved in setting or adjusting ventilator parameters and weaning, but majority of them were involved in suctioning during extubation and some reported to be part of the weaning team. Overall response rate of the physiotherapists involved in setting ventilator parameters was $10 \%$ and respondents involved in weaning was $18 \%$. Whereas in study by Norrenberg \& Vincent (2000) reported that physiotherapists played an active role in the adjustments of mechanical ventilator in $12 \%$ of units, therapists were involved in weaning from mechanical ventilator in $22 \%$ of units. He also found that $25 \%$ therapists were involved in extubation and $46 \%$ were involved in implementation of non invasive mechanical ventilator. Our study showed that only $19.05 \%$ therapists were involved regularly in setting initial mechanical ventilators, $22.62 \%$ were often involved, $28.57 \%$ were occasionally involved and $29.76 \%$ were never involved in setting initial ventilator parameters. This study also showed that $45.24 \%$ respondents were never involved in weaning and only $21.43 \%$ were regularly involved in weaning. This shows that therapists are occasionally involved in other manipulations other then respiratory therapy in NICU and PICU of Punjab.

Conclusion: It was concluded that not all of the patients get routine physiotherapy from the professionally qualified physiotherapists as part of their NICU and PICU stay in hospitals located in Punjab. So, professionally qualified physiotherapists should be a part of multidisciplinary team required for physiotherapy treatment of patients.

Acknowledgment: The authors thank all the physiotherapists who voluntarily participated in this study.

\section{References}

Bertone, N. 1988. The role of physiotherapy in a neonatal intensive care unit. Australian journal of physiotherapy, 34: 27-34.

Bruno, Demont and Claude, Vincon. 2007. Chest physiotherapy using the expiratory flow increase procedure in ventilated newborns: A pilot study. J. Physiother. 93: 12-16.

Farrel, P.M. and Avery, M.E. 1975. Hyaline membrane disease, American Review of Paediatrics, 111: 657-688.

Fernandez, A. and Mondkar, J. A. 1993. Status of neonatal intensive care units in India. Journal of Post Graduate Medicine, 39: 5759.

Jithendra, A Kumar, Arun G. Maiya and Daphne, Pereira. 2007. Role of physiotherapists in intensive care units of India: A multicenter survey. Indian J. Crit. Care Med., 11(4): 198-203.

Lewis, J.A., Lacey, J.L., Henderson-Smart, D.J. 1992. A review of chest physiotherapy in neonatal intensive care units in Australia. $J$. Paediatric Child Health, 28:.297-300.

Manjiri, P. Dighe, Maryann, A., Muckaden, Swati Manerkar, A and Balaji P. Duraisamy. 2011. Is there a Role of Palliative Care in the Neonatal Intensive Care Unit in India? Indian J. Palliat. Care, 17(2): 104-107.

Norrenburg, M. and Vincent, J. 2000. A profile of European intensive care unit physiotherapists. Intensive Care Med., 26: 988-94.

Pronovost, P.J., Dang, D. and Dorman, T. 2001. Intensive Care Unit Nurse Staffing and the 
Risk for Complications after Abdominal Aortic Surgery. American Society of Internal Medicine, 4(5): 199-206.

Robyn, M. Hudson, and Robyn, C. Box. 2003. Neonatal respiratory therapy in the new millennium: Does clinical practice reflect scientific evidence? Australian Journal of Physiotherapy, 49: 269-272.

Vijay Pratap, Singh, Vaishali, Nutan Kamath, Bidita Khandelwal, and R. N. Salhan. 2012. Effect of cardiopulmonary physiotherapy on lung parameters in mechanically ventilated neonates. Int. J. Medicine and Medical Sciences, 4(10): 246-250.

Whitfield, Jonathan M., Peters, Beverly A., Shoemaker, Craig. 2004. A celebration of a century of neonatal care. Conference
Proceedings summary (Dallas: Baylor University Medical Center), 17(3): 255-258. Zach, M.S. and Oberwaldner, B. 1999. Chest physiotherapy. In: Taussig L, Landau L, eds. Textbook of Pediatric Respiratory Medicine, St.Louis, Mosby Inc, 1999; 299-311.

Zach, M.S. and Oberwaldner, B. 1987.Chest Physiotherapy- the mechanical approach to anti-infective therapy in cystic fibrosis. Infection, 5: 381-384.

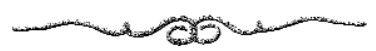

